\title{
Resolving Pulsar Magnetospheres Using Interstellar Scintillation
}

\author{
T. V. Smirnova ${ }^{1}$, V. I. Shishov, and V. M. Malofeev \\ Space Science Center, Lebedev Physical Institute, Moscow, Russia
}

Pulsars are very small objects and to resolve a pulsar magnetosphere you would need an interferometer with a baseline of $14 \mathrm{AU}$ at a frequency $100 \mathrm{MHz}$. If the angular size of the diffraction pattern at the Earth is less than the angular size of pulsar: $\theta_{c}<\theta_{L C}$ then emission regions separated by a light-cylinder radius will produce scintillations that are independent. The interstellar scintillation method presents a unique oppotunity for measuring the angular size of sources with a resolution up to $10^{-8}$ arcsec at meter wavelengths.

There were different attempts to resolve pulsar magnetospheres by the scintillation method (Cordes, Weisberg and Boriakoff, 1983, Wolszcan and Cordes, 1987; Smirnova and Shishov, 1989; Smirnova, 1992). Here we present new observations of 4 pulsars: PSR $0834+06,1133+16,1237+25$ and $1919+21$ at a frequency $102 \mathrm{MHz}$ from which we can see evident decorrelation of the pulsar spectrum with increasing space separation between sources in the pulsar magnetosphere.

For the saturated scintillations for the case of a statistically uniform medium filling all the space between the source and the observer the cross correlation function of the diffraction fluctuation of the intensities of two sources with the space separation $\vec{\rho}_{0}$ is $B_{I}\left(\vec{\rho}_{0}, t\right)=I_{1} I_{2} \exp \left[-\left(\vec{\rho}_{0}-\vec{v}_{s} t\right)^{2} / b^{2}\right]$, where we assumed that the velocity of the pulsar $\vec{v}_{s}$ exceeds all other velocities. From the shift of the maximum of the time crosscorrelation function we can determine the projection of the vector $\vec{\rho}_{0}$ on the velocity vector of the pulsar $\rho_{0, \|}=t_{\max } \cdot v_{s}$ as function of the longitude of the source $l$.

Measurements were made at $102.7 \mathrm{MHz}$ using the Large-Aperture Synthesis Telescope (BSA) of the Lebedev Physics Institute in April and June, 1994. We used a new $128 \times 1.25 \mathrm{KHz}$ multichannel receiver. Pulses were sampled every $5.12 \mathrm{~ms}$ in all 128 channels and data were accumulated during 5 or $20 \mathrm{sec}$.

For studying the decorrelation between pulsa.r spectra at separated longitudes we have calculated a mean cross-correlation functions between the strong pulse ( $S / N \geq 5 \sigma$ ) spectrum taken at the longitude of the leading edge of the pulse profile $l_{0}$ - which was adopted as the reference point - and at all subsequent longitudes $l$. From the falling cross-correlation coefficient between spectra with increasing longitude shift we obtained the absolute value of the baseline $\rho_{0}$ between sources as a function of longitude. The characteristic dimension of the diffraction pattern $b$ we determined using distance of pulsars from catalog and the decorrelation bandwidth as obtained in the work of Malofeev et al (1995). The values of $b, \Delta f_{0}$ and $z$ are listed in Table 1 .

\footnotetext{
${ }^{1}$ Physics Dept., Oberlin College, Oberlin, OH 44074
} 
We also computed the temporal CCF. From the shift of the center of the temporal CCF relative to zero delay we calculated the projection of the vector $\vec{\rho}_{0}$ on the velocity vector of the pulsar: $\rho_{0, \|}(l)$.

In a series of frequency cross-correlation functions the loss of correlation of scintillations with increasing longitude is sharply noticeable. The temporal CCF shows a displacement relative to zero delay for separated longitudes corresponding to different sources, which is markedly obvious from the asymmetry of the CCF with respect to zero delay. The characteristic dimension of the diffraction pattern $b$ we determined using distance of pulsars from catalog and the decorrelation bandwidth as obtained in the work of Malofeev et al (1995). The values of $b, \Delta f_{0}$ and $z$ are listed in Table 1 .

The analysis of our data shows that $\rho_{0, \|}$ is much smaller than $\rho_{0}$. The typical value of $\rho_{0}$ is approximately equal to $b$, while the typical value of $\rho_{0, \|} \simeq$ $(0.1-0.2) b$. This means that the visible distribution of sources is close to that of a straight line, and that the orientation of the straight line is close to that of a normal to the velocity vector of the movement of the pulsar in space. Since the line of sources is oriented along a parallel, that is along a perpendicular to the axis of rotation of the pulsar, we can conclude that the velocity vector of the movement of the pulsar in space is close to the orientation of the rotation axis of the pulsar. Second of all, the dependence of $\rho_{0}$ on $l$ is obviously nonlinear. In the central longitudes of the mean profile a plateau is observed in the dependence of $\rho_{0}$ on $l$.

Using the relations obtained for an approximation to the dipole magnetic field we can estimate the height of the radiating region $h$ as $h=3 \frac{\rho_{0}}{\Delta l}$, where $\Delta l$ is the difference in longitudes of the edges of the averaged pulse profile. The values of $h$ are in Table 1 . We see that the assumption that the magnetic field is dipolar leads to values of $h$ of the order of, or larger than, the radius of the velocity of light cylinder $r_{L C}$. If this is the case we should expect a substantial deviation of the magnetic field structure from that of a dipole. We hope that our observational work will stimulate theoretical work on the structure of the magnetic field at large heights.

\section{References}

Cordes J.M., Weisberg J.M., Boriakoff V., 1983, Ap.J.,268, 370.

Wolszczan A., Cordes J.M., 1987, Ap.J.Lett., 320,L35.

Smirnova T.V., Shishov V.I., 1989, Sov. Pis'ma Astron. Zh., 15, 443.

Smirnova T.V. 1992, Sov. Pis'ma Astron.Zh.,18, 959.

Malofeev V.M., Smirnova T.V., Soin A.G., Shapovalova N.V., 1995, Pis'ma Astron. Zh., 1995, 21, 691.

\begin{tabular}{|c||c|c|c|c|c|c|}
\hline PSR & P, sec & $z, \mathrm{kpc}$ & $\Delta f_{0}, \mathrm{KHz}$ & $b, \mathrm{~cm}$ & $h, \mathrm{~cm}$ & $h / r^{2} C$ \\
\hline \hline $0834+06$ & 1.27 & 0.72 & 2.3 & $4.4 \cdot 10^{8}$ & $5.3 \cdot 10^{9}$ & 0.9 \\
$1133+16$ & 1.19 & 0.27 & 7.2 & $4.7 \cdot 10^{8}$ & $2.0 \cdot 10^{9}$ & 0.4 \\
$1237+25$ & 1.38 & 0.56 & 10.6 & $8.2 \cdot 10^{8}$ & $2.8 \cdot 10^{9}$ & 0.4 \\
$1919+21$ & 1.34 & 0.66 & 5.5 & $6.3 \cdot 10^{8}$ & $9.6 \cdot 10^{9}$ & 1.5 \\
\hline
\end{tabular}

